\title{
Effect of physical training on urinary incontinence: a randomized parallel group trial in nursing homes
}

\author{
This article was published in the following Dove Press journal: \\ Clinical Interventions in Aging \\ 7 February 2012 \\ Number of times this article has been viewed
}

\begin{abstract}
Anne G Vinsnes'
Jorunn L Helbostad ${ }^{2}$

Signe Nyrønning ${ }^{3}$

Gene E Harkless ${ }^{1,4}$

Randi Granbo 5

Arnfinn Seim ${ }^{6}$

'Faculty of Nursing, Sør-Trøndelag University College, ${ }^{2}$ Department of Neuroscience, Norwegian University of Science and Technology, ${ }^{3}$ Søbstad Community Hospital and Teaching Nursing Home, Trondheim, Norway; ${ }^{4}$ University of New Hampshire, College of Health and Social Services, Nursing Faculty, Durham, New Hampshire, USA; ${ }^{5}$ Department of Physiotherapy, Sør-Trøndelag University College, ${ }^{6}$ Department of Public Health and General Practice, Norwegian University of Science and Technology, Trondheim, Norway
\end{abstract}

Correspondence: Anne G Vinsnes Faculty of Nursing, Sør-Trøndelag University College, HiST postmottak, N-7004 Trondheim, Norway Tel +47 73552952

Email anne.g.vinsnes@hist.no
Background: Residents in nursing homes (NHs) are often frail older persons who have impaired physical activity. Urinary incontinence (UI) is a common complaint for residents in NHs. Reduced functional ability and residence in NHs are documented to be risk factors for UI.

Objective: To investigate if an individualized training program designed to improve activity of daily living (ADL) and physical capacity among residents in nursing homes has any impact on UI.

Materials and methods: This randomized controlled trial was a substudy of a Nordic multicenter study. Participants had to be $>65$ years, have stayed in the NH for more than 3 months and in need of assistance in at least one ADL. A total of 98 residents were randomly allocated to either a training group $(n=48)$ or a control group $(n=50)$ after baseline registrations. The training program lasted for 3 months and included accommodated physical activity and ADL training. Personal treatment goals were elicited for each subject. The control group received their usual care. The main outcome measure was UI as measured by a 24-hour pad-weighing test. There was no statistically significant difference between the groups on this measure at baseline $(P=0.15)$. Changes were calculated from baseline to 3 months after the end of the intervention.

Results: Altogether, 68 participants were included in the analysis, 35 in the intervention group and 33 in the control group. The average age was 84.3 years. The 3 months' postintervention adjusted mean difference between groups according to amount of leakage was $191 \mathrm{~g}(P=0.03)$. This result was statistically significant after adjusting for baseline level, age, sex, and functional status. The leakage increased in residents not receiving the experimental intervention, while UI in the training group showed improvement.

Conclusion: The intervention group had significant better results compared with the control group after an individualized training program designed to improve ADL and physical capacity. Further studies are needed to evaluate the effect of a goal-oriented physical training program toward NH residents UI complaints.

Keywords: effect study, nursing homes, residents, physical training program, urinary incontinence

\section{Introduction}

Residents in nursing homes (NHs) could be described as "frail older persons who have impaired physical activity, mobility, muscle strength, cognition, nutrition, and endurance." Urinary incontinence (UI) is related to the severity of comorbidity and functional impairment and may be an early marker of frailty. UI is defined as "involuntary loss of urine that is a social or hygienic problem," and internationally recognized as a risk factor for NH placement. UI is a common complaint amongst 
$\mathrm{NH}$ residents with published prevalence rates ranging from $43 \%$ to $77 \%$. The differences in prevalence can be explained by differences in research method, the way UI is defined, population characteristics, care settings, and care process. ${ }^{2}$ UI presence and its quantification can be reliably determined by using the 24-hour pad-weighting test (24PWT). ${ }^{3}$

In a recent systematic review using data from January 1997 to April 2008, Offermans et al reported that although factors influencing UI are poorly understood, dependency in activities of daily living (ADL), alertness, drugs, and mobility stand out as important risk factors for UI. ${ }^{2}$ Boyington et al found that the prevalence of UI increased over their 4-year study from $65.4 \%$ to $74.3 \%{ }^{4}$ and that all comorbid conditions were higher at postadmission review compared with evaluation at admission. This supported the finding that an increase in UI prevalence is partly due to increasing age and frailty.

In the frail elderly, "a syndromic model" - which describes UI as multiple age-related risk factors both in and outside the lower urinary tract system - interacts synergistically to create UI. ${ }^{1}$ Examples of risk factors in the lower urinary tract in men are prostate hyperplasia, while women are affected by decreases in detrusor contraction strength and urethra closure pressure, lack of estrogen, and pelvic floor dysfunctions. ${ }^{1}$ Factors outside the lower urinary tract that are associated with UI are comorbid medical disease, dementia, falls, dizziness, decreased vision and hearing, neurological and psychiatric disorders, and medication. ${ }^{1}$ The relationship between UI and disability is a complex phenomenon that includes clinical, pharmacological, environmental, and behavioral rehabilitative mechanisms. ${ }^{5}$ However, to develop and test interventions that mediate the onset, severity, or improvement of UI in the frail elderly, several variables may be important.

Abrams et al offer an evidence-based guideline for the assessment and treatment of UI in the frail elderly. ${ }^{6}$ An evidence grade of A was given to performing a functional assessment, including the evaluation of mobility, transfers, manual dexterity and the ability to toilet. ${ }^{6}$ These authors recommend that treatment should depend on underlying causes.

Behavioral therapies such as prompted voiding, timed voiding, and habit training combined with exercises are recommended as UI treatment for persons with both physical and cognitive impairments. Still, there are insufficient data to determine what exactly improves UI. People undergoing treatment trials are often independently mobile and cognitively intact, which is not the case for residents in NHs who frequently experience mobility and cognitive problems. ${ }^{7}$ Studies have found that functional impairment predicts subsequent UI in older adults. Likewise, UI is documented to be a risk factor for functional decline and reduced physical activity and an indicator for frailty. Therefore, given that UI is an indicator for frailty, would an intervention program focused on accommodated physical activity and ADL training decrease UI in frail older people? Functional incidental training (FIT) combines prompted voiding with low-intensity endurance and strength exercises. This approach addresses UI directly by toileting and indirectly by improving physical functioning. It is documented that FIT improves endurance and strength and reduces UI in older residents in NHs. ${ }^{8}$ Still, as behavioral intervention programs have been disappointing with regard to staff compliance, it is interesting to explore what works in the FIT approach; the prompted voiding program or the exercise program?

\section{Objective}

The objective of this study was to investigate if an individualized training program designed to improve ADL and physical capacity among residents in NHs had any impact on UI, as measured by the 24PWT.

\section{Design \\ Study site}

This UI intervention study was a Norwegian one-site substudy of the randomized controlled clinical trial and the Nordic multicenter study Physical Functioning and Activity of Daily Living among Residents in Nursing Homes. ${ }^{9}$ The multicenter study was designed to test the influence of an individually tailored intervention program on physical capacity, degree of dependence on ADL, long-term participation in physical and/or daily activities, and self-rated well-being. ${ }^{9}$ It included five Nordic cities: Stockholm and Uppsala (Sweden); Oslo and Trondheim (Norway); and Odense (Denmark). Altogether, 322 residents from 24 different $\mathrm{NHs}$ in the five cities were included. Results presented in this article are from the substudy in Trondheim, which included four municipal NHs out of the 24 Nordic NHs. ${ }^{10}$ The intervention took place from the fall of 2005 to the spring of 2006.

\section{Subjects}

Inclusion criteria were: age $\geq 65$ years, residence of the $\mathrm{NH}$ for more than 3 months, and need for assistance in at least one ADL. Residents with a life expectancy of $<6$ months were excluded. In total 115 residents from the four NHs in 
Trondheim were invited to take part in the study. The average length of stay was 28.5 (standard deviation [SD] = 34.7) months, and the average mini-mental state examination (MMSE) score was 12.5 (median =12). Four of the eligible residents were not willing to participate, and 13 more residents were excluded from the study due to incomplete UI data at baseline prior to randomization and initiation of the intervention. Hence, 98 residents ( 74 women and 24 men) - 33, 24, 21, and 20 from the four NHs, respectively, were included. The average age at enrollment was 85.7 (SD 8.2) years and women were older than men ( 87.2 versus 81.1 years, $P=0.001)$. The overall prevalence of UI was $80.6 \%$.

The randomization was based on a random numbers list. Consequently, 48 residents were randomized into the intervention group and 50 residents were placed in the control group. Figure 1 displays the accounting of the subjects through the phases of the study.

Sixty-eight residents (51 women and 17 men) were included in the final analysis with 35 (25 women and ten men) in the intervention group and 33 (26 women and seven men) in the control group. The average age at post-test was 84.3 (SD 8.6) years. There was no statistically significant difference between the intervention and control group in age, but women were older than men $(85.7$ versus 80.0 years, $P<0.05)$. The overall prevalence of UI was $89.7 \%$.

\section{Intervention program}

The training program, which included physical activity and ADL training, was individually adjusted for each resident in the intervention group and lasted for 3 months. Personal treatment goals were elicited for each subject. Training in transfer and walking ability, balance, muscle strength, and endurance was offered to individuals and groups, while ADL training was performed when the resident needed help during meals, with personal care, or dressing. Each subject was asked to participate in creative and/or entertaining activities. All staff members on the wards were informed about each resident's treatment goals and offered personal supervision regarding how to provide 'just the right challenge' to the residents. Two physiotherapists and two occupational therapists hired from outside the $\mathrm{NH}$ provided the intervention services. The control group received their usual care from the NH staff based on an individualized care plan.

\section{Outcome measure}

The outcome measure of the 24PWT was quantified prior to the intervention, immediately after the intervention and 3 months after the intervention. Due to insufficient data collection immediately after the intervention, only 3 months of post-intervention data was used in this analysis. No difference between the groups regarding this measure was

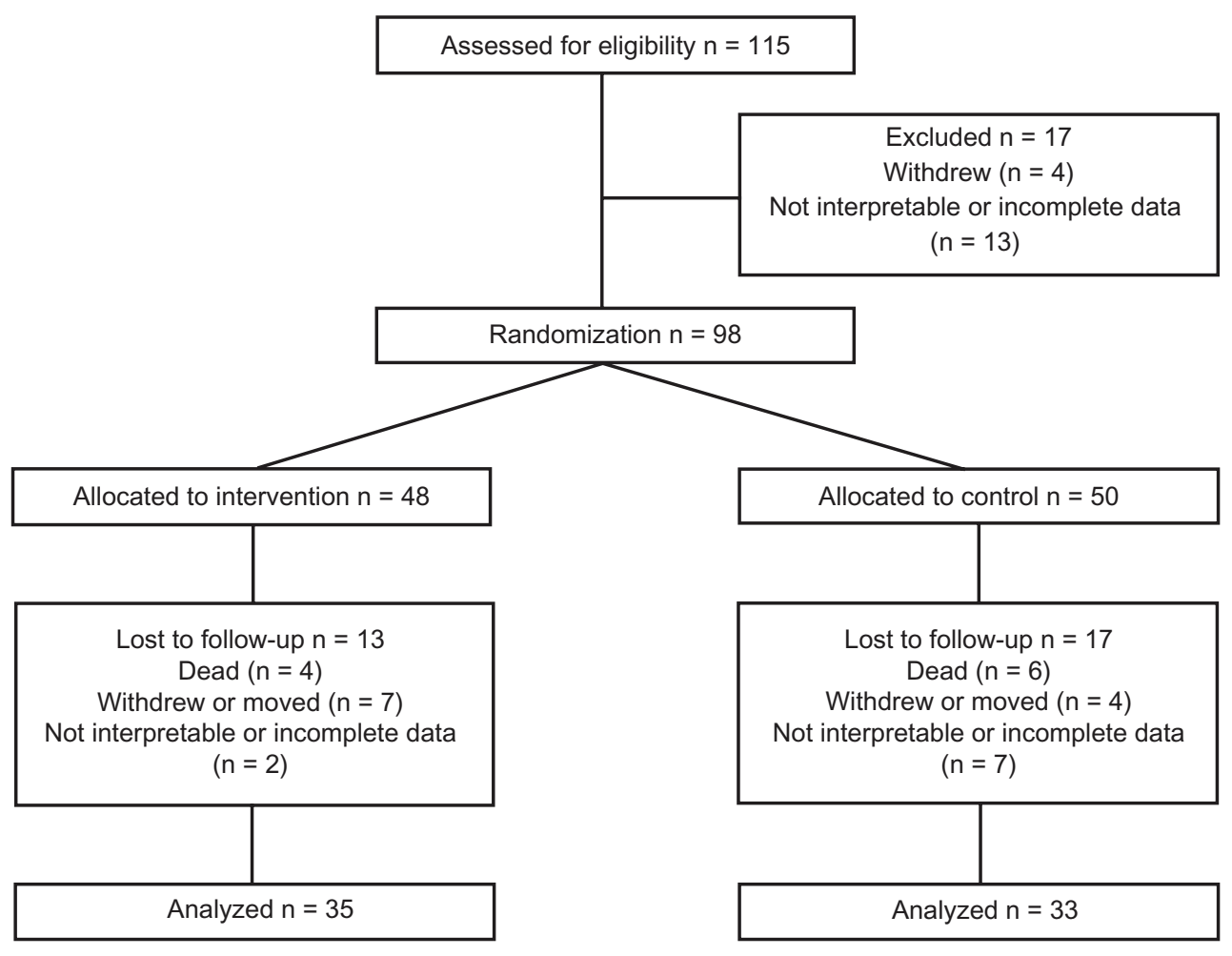

Figure I Flow diagram of the enrolment, intervention allocation, follow-up, and data analysis. 
found at baseline $(P=0.15)$. Furthermore, functional status related to toilet habits was registered, whether the resident was self-reliant, needed a reminder or some help, or was totally dependent on help at the beginning of the study.

\section{Statistical methods}

Descriptive statistics were used to describe the sample and the study measures. Univariate analysis of variance was used to compare differences between the intervention and control group at 3 months post-intervention, adjusting for baseline leakage, age, sex and functional status. The alpha level was set at $P<0.05$. Analyses were also carried out separately among women and men. In the intervention group, multiple linear regression analysis was performed to identify predictors including age, gender, and functional status related to toilet habits dichotomized into need all help versus self-reliant or need some help for the outcome of change in leakage as measured by 24PWT. SPSS software (v 17.0; SPSS, Inc, Chicago, IL) was used for the analyses. Because this study drew on the participants and resources of a larger study, a priori power analysis was not performed. ${ }^{9}$

\section{Ethics}

The study was approved by the Regional Ethics Committee and by the Norwegian Social Science Data Services. Participants gave their written consent to participate. In cases where residents did not have competence to consent, next of kin gave written consent on their behalf (58\% of the included).

\section{Results}

Table 1 shows the distribution of functional status related to toilet habits. Very few residents were self-reliant; 60 out of 68 residents were in need of some help with toileting.

Mean leakage of urine at baseline and 3 months postintervention is shown in Table 2. There was a decrease of leakage in the intervention group while the control group increased in leakage. This difference between groups was statistically significant after adjusting for baseline leakage,

Table I Functional status related to toilet habits and according to group among the 68 included nursing home patients

\begin{tabular}{lll}
\hline Function class & $\begin{array}{l}\text { Intervention } \\
\text { group } \\
(\mathbf{n}=\mathbf{3 5})\end{array}$ & $\begin{array}{l}\text { Control } \\
\text { group } \\
(\mathbf{n}=\mathbf{3 3})\end{array}$ \\
\hline Self-reliant & 4 & 4 \\
Need reminder or some help & 12 & 13 \\
Totally dependent on help & 19 & 16 \\
\hline
\end{tabular}

age, sex, and functional status. Analysis carried out separately among women and men showed the same tendency for both genders.

In the intervention group, the regression analysis showed that increasing age and low functional level (need all help) was statistically significant predictors for improvement after intervention, while being a woman significantly predicted poorer result (Table 3 ).

\section{Discussion}

This study confirms previous findings that in a NH population, UI increases over time. On the other hand, our results signal that this deterioration may be controlled or reversed following an individualized training program designed to improve overall ADL abilities and physical capacity.

Of the 17 residents excluded before randomization, eleven did not have the 24PWT data, and two 24PWTs, although provided, were not interpretable. An explanation for this could be that the 24PWT required a systematic effort from staff members through three shifts. The staff across the 24-hour time period had to understand why and how to complete the test and adhere to the process. An earlier study by Vinsnes et al found that the staff lack knowledge about UI in general and more specifically do not have the skills to investigate UI problems. ${ }^{11}$ Even if the staff believe in the importance of UI assessment and the value of a treatment program, they often fail when it comes to implementation. Hence, lack of staff adherence may explain the uninterpretable or incomplete data at baseline. A combined bladder and drinking chart is a commonly used measure of bladder function, but due to a severe amount of missing data we decided to use the 24PWT as the main outcome measure. In addition, ten residents passed away during the study even though residents with life expectancy less than 6 months were excluded at the beginning. This underscores the fact that the subjects were frail older persons who needed help to perform some or all ADL. The increase of frailty over time is congruent with other studies. $^{4}$

In addition to the passing away of residents, there may be other resident and staff factors leading to withdrawal from the study. One reason for withdrawal might be that the training program required active involvement from the participants. Even if the residents had given their consent to participate, and the program was individually adjusted for each resident, it is reasonable to assume that the extra effort it took to participate may explain why some residents dropped out during the study period. However, this does not explain the drop-out in the control group. A more likely explanation 
Table 2 Daily amount of leakage (grams) at baseline and 3-month post-intervention adjusted for baseline level, age, sex, and functional status

\begin{tabular}{|c|c|c|c|c|c|}
\hline & \multirow{2}{*}{$\begin{array}{l}\text { Intervention } \\
\text { group }(n=35) \\
\text { Mean }(\mathrm{SD})\end{array}$} & \multirow{2}{*}{$\begin{array}{l}\text { Control } \\
\text { Group }(n=33) \\
\text { Mean }(S D)\end{array}$} & \multicolumn{3}{|c|}{ Between group difference } \\
\hline & & & Mean & $95 \% \mathrm{Cl}$ & $P$ value \\
\hline Baseline & $576(465)$ & $424(379)$ & 152 & $-54-358$ & 0.15 \\
\hline 3-months post-intervention & $489(375)$ & $624(454)$ & 135 & $-67-336$ & 0.19 \\
\hline 3-months post-intervention adjusted & 462 & 653 & 191 & $15-368$ & 0.03 \\
\hline
\end{tabular}

Abbreviations: $\mathrm{Cl}$, confidence interval; $\mathrm{SD}$, standard deviation.

is that the 24PWTs demanded too great an effort from staff members on the different day, evening, and night shifts. The incomplete data at baseline, the subsequent need to exclude these participants from our study, and the loss of subjects throughout the study do present a challenge to generalizability. Therefore, we draw our conclusions with caution.

As shown in Table 1, only a few residents were selfreliant in toileting. The larger Nordic study was designed to accept most residents in order to include a case-mix with a wide range of medical diagnoses and functional limitations. ${ }^{9}$ The population in our smaller Norwegian UI study was frail according to the definition of frailty, ${ }^{1}$ which is often the case for residents in NH. ${ }^{7}$ Evidence-based guidelines for the assessment and treatment of $\mathrm{UI}$ in the frail elderly in $\mathrm{NH}$ are available. ${ }^{6}$ The guidelines recommend active screening for UI along with screening for the person's frailty. ${ }^{1}$ The treatment guidelines recommend basing interventions on the resident's preferences for UI care and treatment. Residents' preferences for UI care are of great importance because even cognitively impaired persons can express treatment preferences and treatment satisfaction. ${ }^{1}$

One of the primary findings of our study is that UI differences between groups increased. The 3-month post-intervention adjusted mean difference between groups according to amount of leakage was $191 \mathrm{~g}(P=0.03)$. This result was statistically significant after adjusting for baseline level, age, sex, and functional status. The leakage increased in residents not receiving the experimental intervention of focused physical and ADL training, while UI in the training group showed improvement. This finding challenges us to consider the importance of overall physical functioning in the prevention of deteriorating UI in the frail elderly. In older people, it is documented that functional impairment is a predictor for UI, and that UI is a risk factor for reduced physical activity, functional decline, and frailty. This cause and effect cycle is complicated but this study demonstrates that intervention aimed at overall physical ability improvement may modestly improve UI, as UI may exacerbate functional decline.

For NH residents - given the decline of their medical, physical, and mental state over time - it might be unrealistic to expect a great UI improvement or cure. Another study underlined this when they found that UI increased over time, as did other comorbid conditions. ${ }^{4}$ In light of this, the improvement in our study is noteworthy. However, it may be that our modest improvement in UI reflects the fact that the training program was aimed at all $\mathrm{NH}$ residents and may need to be tailored to have optimal impact on UI in frail elderly residents. A more goal-oriented physical and behavioral training program tailored for residents with UI may lead to a greater decrease of leakage. Overall, the general physical training program was effective in improving UI and, most notably, this improvement was achieved without using the standard behavioral interventions of prompted voiding, timed voiding, and habit training, which are the standard recommended UI treatments in persons with cognitive and physical impairments. FIT combines prompted voiding with low-intensity endurance and strength exercises. There is still a need to further investigate what works; the prompted voiding program

Table 3 Multiple linear regression analysis with change in leakage as dependent variable (intervention group only)

\begin{tabular}{|c|c|c|c|c|}
\hline \multirow[t]{2}{*}{ Covariates } & \multicolumn{2}{|c|}{ Unstandardized coefficients } & \multirow{2}{*}{$\begin{array}{l}\text { Standardized } \\
\text { coefficients } \\
\text { Beta }\end{array}$} & \multirow[t]{2}{*}{$P$ value } \\
\hline & B & Std error & & \\
\hline Age (years) & -16.145 & 7.361 & -0.346 & 0.036 \\
\hline $\operatorname{Sex}($ male $=0$, female $=I)$ & 326.772 & 154.880 & 0.338 & 0.043 \\
\hline Functional level (need little help $=0$, need all help $=I$ ) & -314.326 & 136.445 & -0.359 & 0.028 \\
\hline
\end{tabular}


or the exercise program, or how these programs can be translated into practice. $^{8}$

The regression analysis showed that increasing age was a predictor for improvement in the intervention group. This finding can be explained by the fact that it is the oldest ones who might have 'gone downhill' the most and accordingly have more to gain from physical activity. This was also the case for the residents who were the most physically dependent; they had more to gain from the training program. Being a woman predicted a poorer result. This result was surprising because we relied on the assumption that UI in women is easier to improve by physical training than UI in men. One explanation for this result could be that UI in women has had more attention in general, and therefore they have been more exposed to treatment.

\section{Conclusion}

Living in a $\mathrm{NH}$ is a risk factor for worsening UI. An individualized training program designed to improve ADL and physical capacity may prevent this worsening or reduce UI. Further studies are needed to evaluate the effect of a more tailored goal-oriented physical training program toward residents' UI along with other behavioral interventions. ${ }^{12}$ These trials should also include resident quality of life and cost outcomes.

\section{Acknowledgments}

The study was financed by the Norwegian Ministry of Health and Care Services, the Health Authorities of Mid Norway, supported by grants from Norwegian Nurses Organisation and was carried out as a study under Søbstad Teaching Nursing Home.

\section{Disclosure}

Other than those outlined in the Acknowledgments, the authors declare no conflicts of interest in this work.

\section{References}

1. DuBeau CE, Kuchel GA, Johnson T, Palmer MH, Wagg A. Incontinence in the frail elderly: report from the 4th International Consultation on Incontinence. Neurourol Urodyn. 2010;29(1):165-178.

2. Offermans MPW, Du Moulin MFMT, Hamers JPH, Dassen T, Halfens RJG. Prevalence of urinary incontinence and associated risk factors in nursing home residents: A systematic review. Neurourol Urodyn. 2009;28(4):288-294.

3. Dylewski DA, Jamison MG, Borawski KM, Sherman ND, Amundsen CL, Webster GD. A statistical comparison of pad numbers versus pad weights in the quantification of urinary incontinence. Neurourol Urodyn. 2007;26(1):3-7.

4. Boyington JE, Howard DL, Carter-Edwards L, et al. Differences in resident characteristics and prevalence of urinary incontinence in nursing homes in the southeastern United States. Nurs Res. 2007;56(2): 97-107.

5. Coll-Planas L, Denkinger MD, Nikolaus T. Relationship of urinary incontinence and late-life disability: Implications for clinical work and research in geriatrics. Z Gerontol Geriatr. 2008;41(4):283-290.

6. Abrams P, Andersson KE, Birder L, et al. Fourth International Consultation on Incontinence Recommendations of the International Scientific Committee: Evaluation and treatment of urinary incontinence, pelvic organ prolapse, and fecal incontinence. Neurourol Urodyn. 2010; 29(1):213-240.

7. Leung FW, Schnelle JF. Urinary and fecal incontinence in nursing home residents. Gastroenterol Clin North Am. 2008;37(3):697-707. x.

8. Ouslander JG, Griffiths PC, McConnell E, Riolo L, Kutner M, Schnelle J. Functional incidental training: a randomized, controlled, crossover trial in Veterans Affairs nursing homes. J Am Geriatr Soc. 2005;53(7):1091-1100.

9. Frandin K, Borell L, Gronstedt H, et al. A Nordic multi-center study on physical and daily activities for residents in nursing home settings: design of a randomized, controlled trial. Aging Clin Exp Res. 2009; 21(4-5):314-322.

10. Vinsnes AG, Helbostad JL, Nyrønning S, Granbo R, Seim A. A general physical training programme for residents in nursing homes and the effect on urinary incontinence: a randomized controlled trial. Neurourol Urodyn. 2010;29(6):852-853.

11. Vinsnes AG, Harkless GE, Nyrønning S. Unit-based intervention to improve urinary incontinence in frail elderly. Vard Nord Utveckl Forsk. 2007;27(3):53-56.

12. Palmer MH. Urinary incontinence quality improvement in nursing homes: where have we been? Where are we going? Urol Nurs. 2008; 28(6):439-444, 453.
Clinical Interventions in Aging

\section{Publish your work in this journal}

Clinical Interventions in Aging is an international, peer-reviewed journal focusing on evidence-based reports on the value or lack thereof of treatments intended to prevent or delay the onset of maladaptive correlates of aging in human beings. This journal is indexed on PubMed Central, MedLine, the American Chemical Society's 'Chemical Abstracts Ser-

\section{Dovepress}

vice' (CAS), Scopus and the Elsevier Bibliographic databases. The manuscript management system is completely online and includes a very quick and fair peer-review system, which is all easy to use. Visit $\mathrm{http}: / / \mathrm{www}$.dovepress.com/testimonials.php to read real quotes from published authors. 